\title{
MONOTONICITY OF SOLUTIONS FOR SOME NONLOCAL ELLIPTIC PROBLEMS IN HALF-SPACES
}

\author{
B. BARRIOS, L. DEL PEZZO, J. GARCÍA-MELIÁN \\ AND A. QUAAS
}

\begin{abstract}
In this paper we consider classical solutions $u$ of the semilinear fractional problem $(-\Delta)^{s} u=f(u)$ in $\mathbb{R}_{+}^{N}$ with $u=0$ in $\mathbb{R}^{N} \backslash \mathbb{R}_{+}^{N}$, where $(-\Delta)^{s}, 0<s<1$, stands for the fractional laplacian, $N \geq 2$, $\mathbb{R}_{+}^{N}=\left\{x=\left(x^{\prime}, x_{N}\right) \in \mathbb{R}^{N}: x_{N}>0\right\}$ is the half-space and $f \in C^{1}$ is a given function. With no additional restriction on the function $f$, we show that bounded, nonnegative, nontrivial classical solutions are indeed positive in $\mathbb{R}_{+}^{N}$ and verify

$$
\frac{\partial u}{\partial x_{N}}>0 \text { in } \mathbb{R}_{+}^{N} \text {. }
$$

This is in contrast with previously known results for the local case $s=1$, where nonnegative solutions which are not positive do exist and the monotonicity property above is not known to hold in general even for positive solutions when $f(0)<0$.
\end{abstract}

\section{INTRODUCTION}

The objective of the present paper is to deal with the semilinear problem

$$
\left\{\begin{array}{cl}
(-\Delta)^{s} u=f(u) & \text { in } \mathbb{R}_{+}^{N}, \\
u=0 & \text { in } \mathbb{R}^{N} \backslash \mathbb{R}_{+}^{N},
\end{array}\right.
$$

where $N \geq 2, \mathbb{R}_{+}^{N}=\left\{x=\left(x^{\prime}, x_{N}\right) \in \mathbb{R}^{N}: x_{N}>0\right\}$ is the half-space and $f$ is a $C^{1}$ function. The operator $(-\Delta)^{s}, 0<s<1$, is the well-known fractional laplacian, which is defined on smooth functions as

$$
(-\Delta)^{s} u(x)=\int_{\mathbb{R}^{N}} \frac{u(x)-u(y)}{|x-y|^{N+2 s}} d y,
$$

up to a normalization constant which will be omitted for brevity. The integral in (1.2) has to be understood in the principal value sense, that is, as the limit as $\varepsilon \rightarrow 0$ of the same integral taken in the complement of the ball $B_{\varepsilon}(x)$ of center $x$ and radius $\varepsilon$. Alternatively, this operator can be defined (omitting again the normalization constant) as

$$
(-\Delta)^{s} u(x)=\frac{1}{2} \int_{\mathbb{R}^{N}} \frac{2 u(x)-u(x+y)-u(x-y)}{|y|^{N+2 s}} d y,
$$

where now the integral is absolutely convergent for sufficiently smooth functions.

Problems with nonlocal diffusion related to (1.1) have been intensively studied in the last years, after their appearance when modeling different situations. For instance, anomalous diffusion and quasi-geostrophic flows, turbulence and water waves, molecular dynamics and relativistic quantum 
mechanics of stars (see [9, 15, 17, 37 and references); or mathematical finance (cf. [2, 7, 18]), elasticity problems [34, thin obstacle problem 11], phase transition [1, 10, 36], crystal dislocation [22, 38, and stratified materials 32 .

Our inspiration to study problem (1.1) comes from the local case $s=1$, that is

$$
\left\{\begin{array}{cl}
-\Delta u=f(u) & \text { in } \mathbb{R}_{+}^{N}, \\
u=0 & \text { on } \partial \mathbb{R}_{+}^{N} .
\end{array}\right.
$$

In a seminal series of papers (cf. [3, 4, 5, 6]), Berestycki, Caffarelli and Nirenberg obtained interesting qualitative properties for positive solutions of (1.4) and Lipschitz nonlinearities $f$. Among other results, they showed that if $f(0) \geq 0$, then any positive solution of (1.4) verifies

$$
\frac{\partial u}{\partial x_{N}}>0 \text { in } \mathbb{R}_{+}^{N}
$$

(see [4] or 5]). This property had been shown initially with additional assumptions on both the solutions and the nonlinearities by Dancer in [20] and 21 .

The case $f(0)<0$ is, however, more subtle, and only partial results are known for the moment. See [5], 26] for the case $N=2$, 27] for $N=2,3$ and 19] for dimensions $N \geq 2$. The main reason for this difference is the existence of nonnegative (periodic) solutions which are not strictly positive.

With regard to a similar property as (1.5) for solutions of the nonlocal problem (1.1), only some partial results have been achieved so far, at the best of our knowledge. Let us mention [25] and [30] where monotone, positive nonlinearities where considered, and [16] for the particular case $f(t)=t^{p}$, $p>1$. On the other hand, the very recent preprint [23] analyzes the same question in more general domains, but with a very restricted class of nonlinearities.

Our intention is to show that actually property (1.5) continues to be true for bounded, nonnegative, nontrivial solutions of (1.1) with no additional assumptions placed on the nonlinearity $f$ aside its regularity. This is in striking contrast with problem (1.4), where, as we have remarked, the case $f(0)<0$ remains unsolved in its full generality for the moment.

Throughout this work, we will deal with bounded, classical solutions of (1.1). However, this will not cause a loss in generality, since it is well-known from the regularity theory developed in [35, 13, 14] and bootstrapping that bounded, viscosity solutions of (1.1) are automatically classical. Observe that classical solutions verify $u \in C^{2 s+\beta}\left(\mathbb{R}_{+}^{N}\right)$ for every $\beta \in(0,1)$, and in particular they are seen to be in $C^{1}\left(\mathbb{R}_{+}^{N}\right)$.

Our main result is the following:

Theorem 1. Assume $f \in C^{1}(\mathbb{R})$ and let $u$ be a bounded, nonnegative, nontrivial classical solution of (1.1). Then $u$ is positive and

$$
\frac{\partial u}{\partial x_{N}}>0 \quad \text { in } \mathbb{R}_{+}^{N}
$$


As a consequence of Theorem 1, we can also obtain some Liouville theorems for problem (1.1) with some special nonlinearities.

Theorem 2. Assume $f \in C^{1}(\mathbb{R})$ is such that $f^{\prime}(t)>0$ for $t>0$, and one of the following holds:

(a) $f(0) \neq 0$;

(b) $f(0)=0$ and $f^{\prime}(0)>0$.

Then problem (1.1) does not admit bounded, nonnegative, nontrivial solutions.

An interesting particular case in Theorem 2 is obtained when we set $f(t)=t-1$. In this case the differences between the local version (1.4) and its nonlocal counterpart (1.1) become more evident, since in the former there exists a unique nonnegative solution given by $u(x)=1-\cos x_{N}$ (see [19]), while for the latter we have:

Corollary 3. The problem

$$
\begin{cases}(-\Delta)^{s} u=u-1 & \text { in } \mathbb{R}_{+}^{N}, \\ u=0 & \text { in } \mathbb{R}^{N} \backslash \mathbb{R}_{+}^{N}\end{cases}
$$

does not admit any bounded, nonnegative, nontrivial solution.

It is interesting to remark that Theorem 1 is an important tool to prove other Liouville theorems for bounded solutions of (1.1). Indeed, passing to the limit as $x_{N} \rightarrow+\infty$, we find that such solutions converge to a stable solution of $(-\Delta)^{s} u=f(u)$ in $\mathbb{R}^{N-1}$. Then one can use the nonexistence theorems already available in that situation (cf. for instance [24]).

We conclude the introduction with a couple of comments on our proofs. We use moving planes to show that any nonnegative, bounded, classical solution of (1.1) is monotone in the $x_{N}$ direction. To deal with the moving planes method, we mainly follow the approach in 25. However, instead of representing the solutions of (1.1) with the aid of Green's function in $\mathbb{R}_{+}^{N}$ at the onset, we use it for an adequate truncation related to $u$ and its reflections. This allows us to avoid any monotonicity or sign restriction on $f$.

It is to be noted that at one point in the argument, when it is assumed that the moving of the planes stop somewhere, we need to rule out the existence of solutions which are symmetric with respect to a hyperplane contained in $\mathbb{R}_{+}^{N}$. In the local case, this is only possible under the additional restriction $f(0) \geq 0$, since such solutions do exist if $f(0)<0$. However, we show in the present work that symmetric solutions can not exist with no additional restriction on $f$ (see Theorem 8 below). In our opinion, this is a result of independent interest. Its proof is based on the regularity inherited by symmetry in the strip, which allows to evaluate the equation on the boundary of the half space. Then the nonlocality of the operator implies, loosely speaking, that the interactions between points which are far away in $\mathbb{R}_{+}^{N}$ is too strong and the solution must vanish. This is a remarkable difference with respect to the case $s=1$, where this interaction is not present. 
The rest of the paper is organized as follows: in Section 2 we give some preliminaries and introduce the notation to be used for the moving planes. Section 3 deals with some properties of the Green's function in a half-space taken from [25] and with a different representation in terms of this function. In Section 4, we obtain a nonexistence result for bounded, nonnegative, nontrivial solutions which are symmetric with respect to a hyperplane, and in Section 5 we perform the proof of our main results, Theorems 11 and 2 ,

\section{Some PRELIMINARIES}

In this section, we gather some preliminary properties which will be useful in the forthcoming sections. We notice that, although we are mostly concerned with solutions in the classical sense, other more general concepts of solutions have to be considered at some places in the present work, mainly due to the fact that we work with truncations of the original functions.

Thus, throughout this section we will consider inequalities in the viscosity sense (see [13] for a definition). We begin by considering a version of the maximum principle for the operator $(-\Delta)^{s}$ in unbounded domains, which will be needed below. We believe that this result is new.

Lemma 4. Assume $D \subset \mathbb{R}_{+}^{N}$ is a domain and let $u \in C\left(\mathbb{R}^{N}\right)$ be a bounded function verifying $(-\Delta)^{s} u \geq 0$ in $D$ in the viscosity sense, with $u \geq 0$ in $\mathbb{R}^{N} \backslash D$. Then $u \geq 0$ in $D$.

Proof. First of all observe that the function $\varphi(x)=\left(x_{N}\right)_{+}^{s}$ is $s$-harmonic in $\mathbb{R}_{+}^{N}$, where $\left(x_{N}\right)_{+}$is the function which coincides with $x_{N}$ in $(0,+\infty)$ and vanishes in $(-\infty, 0]$. Indeed, when $x \in \mathbb{R}_{+}^{N}$ :

$$
\begin{aligned}
(-\Delta)^{s} \varphi(x) & =\int_{\mathbb{R}} \frac{\left(x_{N}\right)^{s}-\left(y_{N}\right)_{+}^{s}}{\left|x_{N}-y_{N}\right|^{1+2 s}}\left(\int_{\mathbb{R}^{N-1}} \frac{\left|x_{N}-y_{N}\right|^{1+2 s}}{\left(\left(x_{N}-y_{N}\right)^{2}+\left|x^{\prime}-y^{\prime}\right|^{2}\right)^{\frac{N+2 s}{2}}} d y^{\prime}\right) d y_{N} \\
& =\int_{\mathbb{R}} \frac{\left(x_{N}\right)^{s}-\left(y_{N}\right)_{+}^{s}}{\left|x_{N}-y_{N}\right|^{1+2 s}}\left(\int_{\mathbb{R}^{N-1}} \frac{d z}{\left(1+z^{2}\right)^{\frac{N+2 s}{2}}}\right) d y_{N} \\
& =C \int_{\mathbb{R}} \frac{\left(x_{N}\right)^{s}-\left(y_{N}\right)_{+}^{s}}{\left|x_{N}-y_{N}\right|^{1+2 s}} d y_{N}=0
\end{aligned}
$$

(see for instance the introduction in [12] or Proposition 3.1 in [31]).

Next take $\varepsilon>0$ and consider the function

$$
v_{\varepsilon}(x)=u(x)+\varepsilon\left(x_{N}\right)_{+}^{s}, x \in \mathbb{R}_{+}^{N} .
$$

Since $u$ is bounded, there exists $M>0$ such that $v_{\varepsilon} \geq 0$ if $x_{N} \geq M$. Define the set $D_{M}=D \cap\left\{x \in \mathbb{R}^{N}: 0<x_{N}<M\right\}$. Then, in the viscosity sense,

$$
\left\{\begin{array}{cl}
(-\Delta)^{s} v_{\varepsilon} \geq 0 & \text { in } D_{M}, \\
v_{\varepsilon} \geq 0 & \text { in } \mathbb{R}^{N} \backslash D_{M} .
\end{array}\right.
$$

We are in a position to apply Theorem 2.3 in $\left[30\right.$ ] to conclude that $v_{\varepsilon} \geq 0$ in $\mathbb{R}^{N}$. Letting $\varepsilon \rightarrow 0$, we obtain that $u \geq 0$ in $\mathbb{R}^{N}$. It is worth remarking that, although Theorem 2.3 in [30] is stated for functions which vanish in $\mathbb{R}^{N} \backslash D_{M}$, a careful inspection shows that it is still valid when the involved functions are nonnegative there. 
Before giving our next result, let us introduce some notation related to the method of moving planes. For $\lambda>0$ we denote, as customary:

$$
\begin{aligned}
\Sigma_{\lambda} & :=\left\{x \in \mathbb{R}_{+}^{N}: 0<x_{N}<\lambda\right\} \\
T_{\lambda} & :=\left\{x \in \mathbb{R}^{N}: x_{N}=\lambda\right\} \\
x^{\lambda} & :=\left(x^{\prime}, 2 \lambda-x_{N}\right) \text { (the reflection of } x \text { with respect to } T_{\lambda} \text { ). }
\end{aligned}
$$

If $f$ is a given nonlinearity and $u$ stands for a a bounded, classical nonnegative solution of our problem (1.1) we also set

$$
\begin{aligned}
& u_{\lambda}(x)= \begin{cases}u(x), & x \in \Sigma_{\lambda} \cup\left(\mathbb{R}^{N} \backslash \mathbb{R}_{+}^{N}\right) \\
u\left(x^{\lambda}\right), & x \notin \Sigma_{\lambda} \cup\left(\mathbb{R}^{N} \backslash \mathbb{R}_{+}^{N}\right)\end{cases} \\
& w_{\lambda}(x)=u_{\lambda}(x)-u(x), \quad x \in \mathbb{R}^{N} .
\end{aligned}
$$

Since our ultimate objective is to show that $w_{\lambda}$ is always nonnegative in $\Sigma_{\lambda}$, the following will also be relevant:

$$
\begin{aligned}
& D_{\lambda}=\left\{x \in \Sigma_{\lambda}: w_{\lambda}(x)<0\right\} \\
& W_{\lambda}=\left\{x \in D_{\lambda}: f(u(x))>f\left(u^{\lambda}(x)\right)\right\} \\
& v_{\lambda}=w_{\lambda} \chi_{D_{\lambda}},
\end{aligned}
$$

where $\chi$ will stand throughout the paper for the characteristic function of a set. It is plain that the function $v_{\lambda}$ will only be meaningful when $w_{\lambda}$ is negative somewhere in $\Sigma_{\lambda}$. We next state one of its important properties.

Lemma 5. Assume $w_{\lambda}<0$ somewhere in $\Sigma_{\lambda}$, for some $\lambda>0$. Then,

$$
(-\Delta)^{s} v_{\lambda} \geq\left(f\left(u^{\lambda}\right)-f(u)\right) \chi_{D_{\lambda}} \text { in } \mathbb{R}_{+}^{N},
$$

in the viscosity sense.

Proof. Let us prove first that, when $x \in D_{\lambda}$, (2.2) holds in the classical sense (cf. the proof of Theorem 1.1 in [28]). Denote

$$
z_{\lambda}=w_{\lambda}-v_{\lambda} .
$$

It is clear that in $D_{\lambda}(2.2)$ is equivalent to $(-\Delta)^{s} z_{\lambda} \leq 0$. To prove this last inequality, denote by $E_{\lambda}$ the reflection through the hyperplane $T_{\lambda}$ of $D_{\lambda}$. Using that $z_{\lambda} \equiv 0$ in $D_{\lambda}$ and $z_{\lambda} \geq 0$ in $\Sigma_{\lambda} \backslash D_{\lambda}$, we have for every $x \in D_{\lambda}$ :

$$
\begin{aligned}
(-\Delta)^{s} z_{\lambda}(x) & =-\left(\int_{\Sigma_{\lambda} \backslash D_{\lambda}}+\int_{E_{\lambda}}+\int_{\Sigma_{\lambda}^{c} \backslash E_{\lambda}}\right) \frac{z_{\lambda}(y)}{|x-y|^{N+2 s}} d y \\
& \leq-\left(\int_{\Sigma_{\lambda} \backslash D_{\lambda}}+\int_{\Sigma_{\lambda}^{c} \backslash E_{\lambda}}\right) \frac{z_{\lambda}(y)}{|x-y|^{N+2 s}} d y \\
& =-\int_{\Sigma_{\lambda} \backslash D_{\lambda}} z_{\lambda}(y)\left(\frac{1}{|x-y|^{N+2 s}}-\frac{1}{\left|x-y^{\lambda}\right|^{N+2 s}}\right) d y \leq 0 .
\end{aligned}
$$

Here we have used that $|x-y| \leq\left|x-y^{\lambda}\right|$ when $x \in D_{\lambda}, y \in \Sigma_{\lambda}$, which can be easily checked. Thus (2.2) is proved in $D_{\lambda}$.

On the other hand, when $x \in \mathbb{R}_{+}^{N} \backslash \overline{D_{\lambda}}$, it is immediate that

$$
(-\Delta)^{s} v_{\lambda}(x)=-\int_{D_{\lambda}} \frac{v_{\lambda}(y)}{|x-y|^{N+2 s}} d y \geq 0,
$$


since $v_{\lambda}=0$ in $\mathbb{R}_{+}^{N} \backslash D_{\lambda}$ and $v_{\lambda}<0$ in $D_{\lambda}$. Therefore (2.2) also holds in the classical sense in $\mathbb{R}_{+}^{N} \backslash \overline{D_{\lambda}}$.

However, the function $v_{\lambda}$ needs not be smooth on $\partial D_{\lambda}$, so that it is not to be expected that its fractional laplacian is even well-defined there. But the inequality can be checked in the viscosity sense. To prove this, take $x_{0} \in \partial D_{\lambda}$ and let $\varphi \in C^{\infty}\left(\mathbb{R}^{N}\right)$ be such that $\varphi<v_{\lambda}$ in a reduced neighborhood $\mathcal{U} \backslash\left\{x_{0}\right\}$ of $x_{0}$, with $\varphi\left(x_{0}\right)=v_{\lambda}\left(x_{0}\right)=0$. Then $(-\Delta)^{s} v_{\lambda}\left(x_{0}\right) \geq 0$ means $(-\Delta)^{s} \psi\left(x_{0}\right) \geq 0$, where

$$
\psi(x)= \begin{cases}\varphi(x) & x \in \mathcal{U} \\ v_{\lambda}(x) & x \in \mathbb{R}^{N} \backslash \mathcal{U}\end{cases}
$$

(cf. [13]). The inequality $(-\Delta)^{s} \psi\left(x_{0}\right) \geq 0$ is easily checked since, taking into account that $v_{\lambda} \leq 0$ in $\mathbb{R}^{N}$, so that $\varphi \leq 0$ in $\mathcal{U}$, we deduce

$$
(-\Delta)^{s} \psi\left(x_{0}\right)=-\int_{\mathcal{U}} \frac{\varphi(y)}{|x-y|^{N+2 s}} d y-\int_{\mathbb{R}^{N} \backslash \mathcal{U}} \frac{v_{\lambda}(y)}{|x-y|^{N+2 s}} d y \geq 0,
$$

as was to be shown.

\section{A REPRESENTATION IN THE HALF-SPACE}

In this section, we will show that the function $v_{\lambda}$ verifies an inequality which involves the Green's function in the half-space. As we have already remarked in the Introduction, the representation is rather general and does not impose any additional properties on the nonlinear term $f$. Recall that we are always assuming $0<s<1$.

We introduce the Green's function for $\mathbb{R}_{+}^{N}$ (see [25]). If $x, y \in \mathbb{R}_{+}^{N}$, we let

$$
G_{\infty}^{+}(x, y)=\frac{k_{N}^{s}}{2}|x-y|^{2 s-N} \int_{0}^{\psi_{\infty}^{+}(x, y)} \frac{t^{s-1}}{(t+1)^{\frac{N}{2}}} d t
$$

where

$$
\psi_{\infty}^{+}(x, y)=\frac{4 x_{N} y_{N}}{|x-y|^{2}} .
$$

In (3.1), $k_{N}$ is a positive constant whose actual value is immaterial for us. It is shown in Theorem 3.1 of [25] that if $u \in L^{\infty}\left(\mathbb{R}^{N}\right)$ vanishes outside $\mathbb{R}_{+}^{N}$ and $(-\Delta)^{s} u \in L^{\infty}\left(\mathbb{R}_{+}^{N}\right)$ is nonnegative, then

$$
u(x)=\int_{\mathbb{R}_{+}^{N}} G_{\infty}^{+}(x, y)(-\Delta)^{s} u(y) d y, \quad x \in \mathbb{R}_{+}^{N} .
$$

To avoid the sign restriction just mentioned, we follow a different approach. The information we obtain is slightly weaker, but it suffices for our arguments in the proofs of Section 5. Here is the main result of this section:

Lemma 6. Assume $f$ is locally bounded and let $u$ be a nonnegative, bounded solution of (1.1). Suppose $w_{\lambda}<0$ somewhere in $\Sigma_{\lambda}$, for some $\lambda>0$. Then, for every $x \in W_{\lambda}=\left\{x \in D_{\lambda}: f(u(x))>f\left(u^{\lambda}(x)\right)\right\}$,

$$
v_{\lambda}(x) \geq \int_{W_{\lambda}} G_{\infty}^{+}(x, y)\left(f\left(u^{\lambda}(y)\right)-f(u(y))\right) d y,
$$

where the integral is absolutely convergent. 
In order to prove Lemma 6, we borrow some notation and results from [25]. For $R>0$, define $B_{R}^{+}:=B_{R}\left(R e_{N}\right) \subset \mathbb{R}_{+}^{N}$, where $e_{N}$ stands for the last vector in the canonical basis, and let

$$
G_{R}^{+}(x, y)=\frac{k_{N}^{s}}{2}|x-y|^{2 s-N} \int_{0}^{\psi_{R}^{+}(x, y)} \frac{t^{s-1}}{(t+1)^{\frac{N}{2}}} d t,
$$

with

$$
\psi_{R}^{+}(x, y)=\frac{\left(R^{2}-\left|x-R e_{N}\right|^{2}\right)\left(R^{2}-\left|y-R e_{N}\right|^{2}\right)}{R^{2}|x-y|^{2}},
$$

be the Green's function in the ball $B_{R}^{+}$. We also introduce

$$
\Gamma_{R}^{+}(x, y)=C_{N, s}\left(\frac{R^{2}-\left|x-R e_{N}\right|^{2}}{\left|y-R e_{N}\right|^{2}-R^{2}}\right)^{s}|x-y|^{-N},
$$

the Poisson kernel for the same ball (cf. 8 for some properties of both functions). According to Corollary 2.9 in [25], if $h_{R}$ is the unique solution of the Dirichlet problem

$$
\left\{\begin{array}{cl}
(-\Delta)^{s} h=g_{1} & \text { in } B_{R}^{+}, \\
h=g_{2} & \text { in } \mathbb{R}^{N} \backslash B_{R}^{+},
\end{array}\right.
$$

where $g_{1} \in L^{\infty}\left(B_{R}^{+}\right)$and $g_{2} \in L^{\infty}\left(\mathbb{R}^{N} \backslash B_{R}^{+}\right)$, then we can write:

$$
h_{R}(x)=\int_{B_{R}^{+}} G_{R}^{+}(x, y) g_{1}(y) d y+\int_{\mathbb{R}^{N} \backslash B_{R}^{+}} \Gamma_{R}^{+}(x, y) g_{2}(y) d y .
$$

Regarding this representation, it is to be noted that, when $g_{2} \in L^{\infty}\left(\mathbb{R}^{N}\right)$, as a consequence of equation (3.7) in [25], then

$$
\lim _{R \rightarrow+\infty} \int_{\mathbb{R}^{N} \backslash B_{R}^{+}} \Gamma_{R}^{+}(x, y) g_{2}(y) d y=0
$$

for every $x \in \mathbb{R}_{+}^{N}$. The following properties of Green's function will be used in our proof of Lemma 6 and in the proof of Theorem 1 in Section 5.

Lemma 7. Fix $R_{0}>0$. Then the functions $G_{R}^{+}(x, y)$ are nondecreasing with respect to $R$ in $B_{R_{0}}^{+} \times B_{R_{0}}^{+}$if $R>R_{0}$ and verify

$$
G_{R}^{+} \rightarrow G_{\infty}^{+} \quad \text { in } B_{R_{0}}^{+} \times B_{R_{0}}^{+} \text {as } R \rightarrow+\infty .
$$

Moreover, for every $\lambda>0$, there exists $C=C(N, s, \lambda)$ such that

$$
G_{\infty}^{+}(x, y) \leq C \min \left\{|x-y|^{2 s-N},|x-y|^{-N}\right\} \quad \text { for } x, y \in \Sigma_{\lambda} .
$$

In addition, the function $G_{\infty}^{+}(x, y)$ enjoys the following properties:

(a) If $\left\{x_{n}\right\}$ is a bounded sequence, then for every $\lambda>0$

$$
\lim _{R \rightarrow+\infty} \int_{\Sigma_{\lambda} \cap B_{R}^{c}} G_{\infty}^{+}\left(x_{n}, y\right) d y=0,
$$

uniformly in $n$.

(b) If $\lambda>0$ and $\left\{x_{n}\right\}$ is a bounded sequence, then for every $R>0$ there exists a positive constant $C$ such that

$$
\int_{\Sigma_{\lambda} \cap B_{R}} G_{\infty}^{+}\left(x_{n}, y\right) d y \leq C \quad \text { for every } n \in \mathbb{N} .
$$


(c) For every $\lambda>0$ and $x_{0} \in \partial \mathbb{R}_{+}^{N}$, we have

$$
\lim _{x \rightarrow x_{0}} \int_{\Sigma_{\lambda}} G_{\infty}^{+}(x, y) d y=0 .
$$

Sketch of proof. The statements about monotonicity and convergence of $G_{R}^{+}$ are a consequence of Lemma 3.2 in 25]. The estimates (3.4) follow because of Lemma 4.1 there.

Parts (a) and (b) are a direct consequence of (3.4), while for the proof of (c), we only have to notice that, if $h \in C\left(\mathbb{R}^{N}\right)$ is the unique solution of

$$
\left\{\begin{array}{cl}
(-\Delta)^{s} h=\chi_{\Sigma_{\lambda}} & \text { in } \mathbb{R}_{+}^{N}, \\
h=0 & \text { in } \mathbb{R}^{N} \backslash \mathbb{R}_{+}^{N},
\end{array}\right.
$$

then by Theorem 3.1 in [25] we have

$$
h(x)=\int_{\Sigma_{\lambda}} G_{\infty}^{+}(x, y) d y,
$$

and the proof follows because of the continuity of $h$ up to the boundary of $\mathbb{R}_{+}^{N}$.

We can now proceed to the proof of Lemma 6 ,

Proof of Lemma 6. We start by observing that, by Lemma 5

$$
(-\Delta)^{s} v_{\lambda} \geq\left(f\left(u^{\lambda}\right)-f(u)\right) \chi_{D_{\lambda}} \text { in } \mathbb{R}_{+}^{N} .
$$

Consider the balls $B_{R}^{+}$introduced before and denote by $h_{R}$ the unique solution of the problem

$$
\left\{\begin{array}{cl}
(-\Delta)^{s} h=\left(f\left(u^{\lambda}\right)-f(u)\right) \chi_{D_{\lambda}} & \text { in } B_{R}^{+}, \\
h=v_{\lambda} & \text { in } \mathbb{R}^{N} \backslash B_{R}^{+} .
\end{array}\right.
$$

It is clear by (3.5) and the maximum principle that $v_{\lambda} \geq h_{R}$ in $B_{R}^{+}$. Therefore, according to (3.2), we may write

$$
\begin{aligned}
v_{\lambda}(x) \geq h_{R}(x) & =\int_{B_{R}^{+} \cap D_{\lambda}} G_{R}^{+}(x, y)\left(f\left(u^{\lambda}(y)\right)-f(u(y))\right) d y \\
& +\int_{\mathbb{R}_{+}^{N} \backslash B_{R}^{+}} \Gamma_{R}^{+}(x, y) v_{\lambda}(y) d y .
\end{aligned}
$$

Our intention is to pass to the limit in (3.7). Notice that, since $v_{\lambda}$ is bounded, we have by (3.3) that the last integral converges to zero as $R \rightarrow+\infty$.

On the other hand, we obtain from Lemma 7 that $G_{R}^{+}$is nondecreasing as a function of $R$ and, for fixed $x \in \Sigma_{\lambda}$

$$
G_{R}^{+}(x, y) \leq G_{\infty}^{+}(x, y) \leq C \min \left\{|x-y|^{-N+2 s},|x-y|^{-N}\right\} \in L^{1}\left(\Sigma_{\lambda}\right),
$$

as a function of $y$. Therefore, letting $R \rightarrow+\infty$ in (3.7) and using dominated convergence we arrive at

$$
\begin{aligned}
v_{\lambda}(x) & \geq \int_{D_{\lambda}} G_{\infty}^{+}(x, y)\left(f\left(u^{\lambda}(y)\right)-f(u(y))\right) d y \\
& \geq \int_{W_{\lambda}} G_{\infty}^{+}(x, y)\left(f\left(u^{\lambda}(y)\right)-f(u(y))\right) d y,
\end{aligned}
$$


as was to be proved.

Remark 1. Similar results as the ones given in this section follow easily in other half-spaces by means of a simple change of variables. For instance, in $H:=\left\{x \in \mathbb{R}^{N}: x_{N}<\lambda\right\}$, the Green's function is given by

$$
G(x ; y)=G_{\infty}^{+}\left(x^{\prime}, \lambda-x_{N} ; y^{\prime}, \lambda-y_{N}\right), \quad x, y \in H,
$$

and similar properties as those given in Lemma 6 are obtained at once.

\section{A nonexistence theOREM}

In this section we will state and prove a nonexistence result for nonnegative solutions of (1.1) which are symmetric with respect to a hyperplane. This result is fairly important in the moving planes argument, and it is the reason why the case $f(0)<0$ can be included in our proofs, in contrast with the local case $s=1$. We believe it is interesting in its own right.

It is to be noted that, when the nonlinearity verifies $f(0) \geq 0$, the nonexistence of these symmetric solutions is a direct consequence of the strong maximum principle. The proof we give, however, covers also this case. Observe that next theorem holds with minimal hypotheses on $f$.

Theorem 8. Assume $f$ is continuous at zero and let $u \in C^{2 s+\beta}\left(\mathbb{R}_{+}^{N}\right)$ $(0<\beta<1)$ be a bounded, nonnegative, classical solution of (1.1) which is symmetric with respect to $T_{\lambda}$ in $\Sigma_{2 \lambda}$ for some $\lambda>0$, that is

$$
u\left(x^{\prime}, 2 \lambda-x_{N}\right)=u\left(x^{\prime}, x_{N}\right), \quad x \in \Sigma_{2 \lambda} .
$$

Then $f(0)=0$ and $u \equiv 0$ in $\mathbb{R}^{N}$.

Proof. We begin by showing that $u$ verifies the equation at $x=0$, that is,

$$
\int_{\mathbb{R}_{+}^{N}} \frac{u(y)}{|y|^{N+2 s}} d y=-f(0) .
$$

To see this, we first observe that with no loss of generality we may assume that $\beta$ is restricted to satisfy $2 s+\beta<2$. Thus the symmetry of $u$ implies that the same regularity holds up to the boundary of $\mathbb{R}_{+}^{N}$, since necessarily $u$ and $\nabla u$ vanish there. Therefore $u \in C^{2 s+\beta}\left(\mathbb{R}^{N}\right)$.

Take an arbitrary sequence $\left\{x_{n}\right\} \subset \Sigma_{2 \lambda}$ with $x_{n} \rightarrow 0$. Evaluating the equation at $x_{n}$, but using expression (1.3) for $(-\Delta)^{s}$, we see that

$$
f\left(u\left(x_{n}\right)\right)=\frac{1}{2} \int_{\mathbb{R}^{N}} \frac{2 u\left(x_{n}\right)-u\left(x_{n}+y\right)-u\left(x_{n}-y\right)}{|y|^{N+2 s}} d y .
$$

Now we have to distinguish between the cases $0<s<\frac{1}{2}$ and $\frac{1}{2} \leq s<1$. In the former case, assuming $\beta$ is such that $2 s+\beta<1$, we deduce from the regularity of $u$ that for sufficiently large $n$ :

$$
\left|u\left(x_{n}\right)-u\left(x_{n}+y\right)\right| \leq C|y|^{2 s+\beta} \quad \text { whenever }|y| \leq 1,
$$

for some positive constant $C$. In the latter, if $\beta$ is such that $2 s+\beta<2$, the regularity implies, also for large enough $n$

$$
\left|u\left(x_{n}\right)-u\left(x_{n}+y\right)-u\left(x_{n}-y\right)\right| \leq C|y|^{2 s+\beta} \quad \text { if }|y| \leq 1 .
$$


On the other hand, for $|y| \geq 1$,

$$
\left|\frac{u\left(x_{n}\right)-u\left(x_{n}+y\right)-u\left(x_{n}-y\right)}{|y|^{N+2 s}}\right| \leq 3\|u\|_{L^{\infty}\left(\mathbb{R}_{+}^{N}\right)}|y|^{-N+2 s} .
$$

Inequalities (4.3), (4.4) and (4.5) show that the integrand in (4.2) is bounded in absolute value by a function which is in $L^{1}\left(\mathbb{R}^{N}\right)$. Therefore, we may pass to the limit in (4.2) with the aid of dominated convergence to arrive at

$$
f(0)=-\frac{1}{2} \int_{\mathbb{R}^{N}} \frac{u(y)+u(-y)}{|y|^{N+2 s}} d y,
$$

which is equivalent to (4.1).

By evaluating the equation at $x=2 \lambda e_{N}$, using the fact that $u\left(2 \lambda e_{N}\right)=0$ by symmetry, we deduce from (4.1) that

$$
\int_{\mathbb{R}_{+}^{N}} \frac{u(y)}{|y|^{N+2 s}} d y=\int_{\mathbb{R}_{+}^{N}} \frac{u(y)}{\left|2 \lambda e_{N}-y\right|^{N+2 s}} d y .
$$

Next, we split the second integral in two parts and use the symmetry of $u$ in $\Sigma_{2 \lambda}$ to have:

$$
\begin{aligned}
\int_{\mathbb{R}_{+}^{N}} \frac{u(y)}{\left|2 \lambda e_{N}-y\right|^{N+2 s}} d y & =\left(\int_{\Sigma_{2 \lambda}}+\int_{\mathbb{R}_{+}^{N} \backslash \Sigma_{2 \lambda}}\right) \frac{u(y)}{\left|2 \lambda e_{N}-y\right|^{N+2 s}} d y \\
& =\int_{\Sigma_{2 \lambda}} \frac{u(z)}{|z|^{N+2 s}} d z+\int_{\mathbb{R}_{+}^{N} \backslash \Sigma_{2 \lambda}} \frac{u(y)}{\left|2 \lambda e_{N}-y\right|^{N+2 s}} d y .
\end{aligned}
$$

Hence, from (4.6) we see that

$$
\int_{\mathbb{R}_{+}^{N} \backslash \Sigma_{2 \lambda}} \frac{u(y)}{|y|^{N+2 s}} d y=\int_{\mathbb{R}_{+}^{N} \backslash \Sigma_{2 \lambda}} \frac{u(y)}{\left|2 \lambda e_{N}-y\right|^{N+2 s}} d y .
$$

Taking into account that $u \geq 0$ and $\left|2 \lambda e_{N}-y\right| \leq|y|$ for $y \in \mathbb{R}_{+}^{N} \backslash \Sigma_{2 \lambda}$, we deduce $u \equiv 0$ in $\mathbb{R}_{+}^{N} \backslash \Sigma_{2 \lambda}$.

Using this information and evaluating the equation at points $x \in \mathbb{R}_{+}^{N} \backslash \Sigma_{2 \lambda}$, we obtain

$$
\int_{\Sigma_{2 \lambda}} \frac{u(y)}{|x-y|^{N+2 s}} d y=-f(0) .
$$

Now observe that the integral above is a smooth function of $x$ if, say, $x_{N} \geq$ $2 \lambda+1$, since the integrand is uniformly bounded and the integral is uniformly convergent at infinity when $x$ belongs to a compact set. Therefore, we are allowed to differentiate (4.7) with respect to $x_{N}$ to get:

$$
\int_{\Sigma_{2 \lambda}} \frac{u(y)\left(x_{N}-y_{N}\right)}{|x-y|^{N+2 s+2}} d y=0 .
$$

However, $x_{N}-y_{N} \geq x_{N}-2 \lambda \geq 1$ for $y \in \Sigma_{2 \lambda}$ and the chosen values of $x$, so that the integrand is nonnegative and this gives $u \equiv 0$ in $\Sigma_{2 \lambda}$, therefore in $\mathbb{R}^{N}$. It is clear that this can only happen when $f(0)=0$, and the proof is concluded. 


\section{Proof of the MAin Results}

In this final section we will prove our main contributions, Theorems 1 and 2. The proof of Corollary 3 will not be given, since it is an immediate consequence of Theorem 2 .

Proof of Theorem 1. We have already said that the proof is an application of the method of moving planes, as used in [30] and [25], but with some significant changes. In particular, we remark that we work with some truncations of the original functions, so we are led to the use of inequalities in the viscosity sense and Lemma 6. We also need at some point the nonexistence result given by Theorem 8 ,

We follow the notation introduced in Section 2.

Step 1. $w_{\lambda} \geq 0$ in $\Sigma_{\lambda}$ if $\lambda>0$ is small enough.

To prove this, assume for a contradiction that $D_{\lambda}$ is not empty if $\lambda$ is small. Since $u$ is bounded and $f$ is $C^{1}$, there exists a constant $L$ such that $f\left(u^{\lambda}\right)-f(u) \geq-L\left|u^{\lambda}-u\right|$. Therefore, using Lemma 5 we have

$$
(-\Delta)^{s} v_{\lambda} \geq f\left(u^{\lambda}\right)-f(u) \geq-L\left|u^{\lambda}-u\right|=L v_{\lambda} \quad \text { in } D_{\lambda} .
$$

By Theorem 2.4 in [30] we obtain $v_{\lambda} \geq 0$ in $D_{\lambda}$ when $\lambda$ is small enough, which is a contradiction. Therefore, $D_{\lambda}=\emptyset$ for small $\lambda$ and this shows the claim.

Step 2. Setting

$$
\lambda^{*}=\sup \left\{\lambda>0: w_{\mu} \geq 0 \text { in } \Sigma_{\mu} \text { for every } \mu \in(0, \lambda)\right\},
$$

we have $\lambda^{*}=+\infty$.

Assume again for a contradiction that $\lambda^{*}<+\infty$. Then there exists a sequence $\left\{\lambda_{j}\right\}$ of values such that $\lambda_{j}>\lambda^{*}$ for every $j$ and $\lambda_{j} \rightarrow \lambda^{*}$ as $j \rightarrow+\infty$, with $w_{\lambda_{j}}$ negative somewhere in $\Sigma_{\lambda_{j}}$. Consider the sets

$$
D_{j}=\left\{x \in \Sigma_{\lambda_{j}}: w_{\lambda_{j}}(x)<0\right\}
$$

and

$$
W_{j}=\left\{x \in D_{j}: f(u(x))>f\left(u^{\lambda_{j}}(x)\right)\right\} .
$$

By the choice of $\lambda_{j}$, the sets $D_{j}$ are nonempty for every $j$. We claim that the same is true for $W_{j}$. Indeed, if we had $W_{j}=\emptyset$, then $f\left(u^{\lambda_{j}}\right) \geq f(u)$ in $D_{j}$. Hence

$$
(-\Delta)^{s} v_{\lambda_{j}} \geq 0 \text { in } D_{j}
$$

By Lemma 4 we obtain $v_{\lambda_{j}} \geq 0$ in $D_{j}$, which is not possible. Therefore $W_{j} \neq \emptyset$. Thus it is possible to choose points $x_{j} \in W_{j}$ such that

$$
v_{\lambda_{j}}\left(x_{j}\right) \leq-\frac{1}{2}\left\|v_{\lambda_{j}}\right\|_{L^{\infty}\left(W_{j}\right)}
$$

and we can define the functions

$$
\widetilde{u}_{j}(x)=u\left(x^{\prime}+x_{j}^{\prime}, x_{N}\right), \quad x \in \mathbb{R}_{+}^{N} .
$$


It is easily seen that $\widetilde{u}_{j}$ is a solution of (1.1), verifying $\left\|\widetilde{u}_{j}\right\|_{L^{\infty}\left(\mathbb{R}_{+}^{N}\right)}=$ $\|u\|_{L^{\infty}\left(\mathbb{R}_{+}^{N}\right)}$. It is then standard, with the use of regularity theory, AscoliArzelá's theorem and a diagonal argument, that for some subsequence

$$
\widetilde{u}_{j} \rightarrow \bar{u}
$$

locally uniformly in $\mathbb{R}^{N}$, where $\bar{u}$ is a nonnegative solution of (1.1). We may also assume that $x_{j, N} \rightarrow x_{0} \in\left[0, \lambda^{*}\right]$. Now three cases are possible:

(a) $\bar{u} \not \equiv 0, x_{0} \in\left(0, \lambda^{*}\right)$;

(b) $\bar{u} \not \equiv 0, x_{0}=0$ or $x_{0}=\lambda^{*}$;

(c) $\bar{u} \equiv 0$.

Before dealing with each of this cases, let us introduce some notation related to the functions $\widetilde{u}_{j}$. Let:

$$
\begin{aligned}
& \widetilde{w}_{\lambda_{j}}(x)=\widetilde{u}_{j}\left(x^{\lambda_{j}}\right)-\widetilde{u}_{j}(x), \quad x \in \Sigma_{\lambda_{j}}, \\
& \widetilde{D}_{j}=\left\{x \in \Sigma_{\lambda_{j}}: \widetilde{w}_{j}(x)<0\right\}, \\
& \widetilde{v}_{\lambda_{j}}(x)=\widetilde{w}_{\lambda_{j}}(x) \chi_{\widetilde{D}_{j}}(x), \quad x \in \Sigma_{\lambda_{j}}, \\
& \widetilde{W}_{j}=\left\{x \in \widetilde{D}_{j}: f\left(\widetilde{u}_{j}(x)\right)>f\left(\widetilde{u}_{j}\left(x_{j}^{\lambda}\right)\right)\right\}
\end{aligned}
$$

(observe that $\widetilde{D}_{j}$ and $\widetilde{W}_{j}$ are nothing more than translations of $D_{j}$ and $W_{j}$, respectively). Denote also $z_{j}=\left(0, x_{j, N}\right), z_{0}=\left(0, x_{0}\right)$. By our choice of $x_{j}$ in (5.1) above, since it follows that $\widetilde{w}_{\lambda_{j}}\left(z_{j}\right)<0$ and $f\left(\widetilde{u}_{j}\left(z_{j}\right)\right)>f\left(\widetilde{u}_{j}\left(z_{j}^{\lambda_{j}}\right)\right)$, we deduce that $z_{j} \in \widetilde{W}_{j}$. Moreover we also get

$$
\widetilde{v}_{\lambda_{j}}\left(z_{j}\right) \leq-\frac{1}{2}\left\|\widetilde{v}_{\lambda_{j}}\right\|_{L^{\infty}\left(\widetilde{W}_{j}\right)} .
$$

Now consider in turn each one of the cases (a), (b) and (c).

In case (a), we see from $\widetilde{u}_{j}\left(x^{\lambda^{*}}\right) \geq \widetilde{u}_{j}(x)$ in $\Sigma_{\lambda^{*}}$ that $\bar{u}\left(x^{\lambda^{*}}\right) \geq \bar{u}(x)$ in $\Sigma_{\lambda^{*}}$. Moreover, since $\widetilde{u}\left(z_{j}^{\lambda_{j}}\right)<\widetilde{u}\left(z_{j}\right)$, we also have $\bar{u}\left(z_{0}^{\lambda^{*}}\right)=\bar{u}\left(z_{0}\right)$. Let us see that this implies $\bar{u}\left(x^{\lambda^{*}}\right) \equiv \bar{u}(x)$ in $\mathbb{R}^{N}$. Indeed, arguing as in the proof of Lemma 5 and denoting $\bar{w}_{\lambda^{*}}=\bar{u}^{\lambda^{*}}-\bar{u}$, we obtain

$$
\begin{aligned}
0 & =f\left(\bar{u}^{\lambda^{*}}\left(z_{0}\right)\right)-f\left(\bar{u}\left(z_{0}\right)\right)=(-\Delta)^{s} \bar{w}_{\lambda^{*}}\left(z_{0}\right) \\
& =-\int_{\Sigma_{\lambda^{*} \cup \mathbb{R}_{-}^{N}}} \bar{w}_{\lambda^{*}}(y)\left(\frac{1}{\left|z_{0}-y\right|^{N+2 s}}-\frac{1}{\left|z_{0}-y^{\lambda}\right|^{N+2 s}}\right) d y,
\end{aligned}
$$

which implies $\bar{w}_{\lambda^{*}} \equiv 0$ in $\mathbb{R}^{N}$, since $\bar{w}_{\lambda^{*}} \geq 0$ in $\Sigma_{\lambda^{*}} \cup \mathbb{R}_{-}^{N}$ and $\left|z_{0}-y\right| \leq$ $\left|z_{0}-y^{\lambda}\right|$ for every $y \in \Sigma_{\lambda^{*}} \cup \mathbb{R}_{-}^{N}$.

This means that $\bar{u}$ is symmetric with respect to $T_{\lambda^{*}}$, so that Theorem 8 implies $\bar{u} \equiv 0$, a contradiction. 
As for case (b), assume $x_{0}=0$. We deduce from Lemma 6 and the choice of the points $z_{j}$ :

$$
\begin{aligned}
\frac{1}{2}\left\|\widetilde{v}_{\lambda_{j}}\right\|_{L^{\infty}\left(\widetilde{W}_{j}\right)} \leq-\widetilde{v}_{\lambda_{j}}\left(z_{j}\right) & \leq L\left\|\widetilde{v}_{\lambda_{j}}\right\|_{L^{\infty}\left(\widetilde{W}_{j}\right)} \int_{\widetilde{W}_{j}} G_{\infty}^{+}\left(z_{j}, y\right) d y \\
& \leq L\left\|\widetilde{v}_{\lambda_{j}}\right\|_{L^{\infty}\left(\widetilde{W}_{j}\right)} \int_{\Sigma_{\lambda^{*}+1}} G_{\infty}^{+}\left(z_{j}, y\right) d y
\end{aligned}
$$

where $L$ denotes a bound for the derivative of $f$ in $\left[0,\|u\|_{L^{\infty}\left(\mathbb{R}_{+}^{N}\right)}\right]$. By Lemma 7. part (c), the last integral converges to zero since $z_{j} \rightarrow 0 \in \partial \mathbb{R}_{+}^{N}$. We deduce that $\widetilde{v}_{\lambda_{j}}=0$ in $\widetilde{W}_{j}$ when $j$ is large enough, a contradiction.

When $x_{0}=\lambda^{*}$, a similar contradiction is reached. The only difference is that one now works with the Green's function in the half-space $\left\{x \in \mathbb{R}^{N}\right.$ : $\left.x_{N}<\lambda_{j}\right\}$ (see Remark 1).

Finally, we consider case (c). This case can only arise when $f(0)=0$ and the proof is different depending on the sign of $f^{\prime}(0)$. We begin by assuming that $f^{\prime}(0)>0$.

In what follows, we denote by $\lambda_{1}(\Omega)$ the principal eigenvalue of $(-\Delta)^{s}$ in $\Omega$ under Dirichlet boundary conditions (cf. Proposition 9 in [33]). If we take a ball $B_{R}$ with arbitrary center and radius $R$ then it can be seen by means of a simple scaling that

$$
\lambda_{1}\left(B_{R}\right)=\frac{\lambda_{1}\left(B_{1}\right)}{R^{2 s}} \rightarrow 0 \quad \text { as } R \rightarrow+\infty .
$$

Thus it is possible to select a ball $B \subset \subset \mathbb{R}_{+}^{N}$ with the property that

$$
\lambda_{1}(B)<\frac{1}{2} f^{\prime}(0) .
$$

Since $\widetilde{u}_{j} \rightarrow 0$ uniformly in $B$, we deduce

$$
(-\Delta)^{s} \widetilde{u}_{j}=\frac{f\left(\widetilde{u}_{j}\right)}{\widetilde{u}_{j}} \widetilde{u}_{j} \geq \frac{1}{2} f^{\prime}(0) \widetilde{u}_{j} \quad \text { in } B
$$

if $j$ is large enough. By Theorem 1.1 in [29], (5.4) implies the opposite inequality in (5.3), which is a contradiction.

Hence to conclude the proof only the case $f^{\prime}(0) \leq 0$ needs to be dealt with. Using the mean value theorem, we may write, for $y \in \widetilde{W}_{j}$ :

$$
f\left(\widetilde{u}_{j}^{\lambda_{j}}(y)\right)-f\left(\widetilde{u}_{j}(y)\right)=f^{\prime}\left(\xi_{j}(y)\right) \widetilde{v}_{j}(y),
$$

where $\xi_{j}(y)$ is an intermediate value between $\widetilde{u}_{j}(y)$ and $\widetilde{u}_{j}^{\lambda_{j}}(y)$. Observe that $\xi_{j} \rightarrow 0$ uniformly on compact sets of $\mathbb{R}_{+}^{N}$ while $f^{\prime}\left(\xi_{j}\right) \geq 0$. By Lemma 6 and (5.2), we see that

$$
\frac{1}{2}\left\|\widetilde{v}_{\lambda_{j}}\right\|_{L^{\infty}\left(\widetilde{W}_{j}\right)} \leq\left\|\widetilde{v}_{\lambda_{j}}\right\|_{L^{\infty}\left(\widetilde{W}_{j}\right)} \int_{\Sigma_{\lambda^{*}+1}} G_{\infty}^{+}\left(z_{j}, y\right) f^{\prime}\left(\xi_{j}(y)\right) d y .
$$


Now choose $R>0$ and split the integral in (5.5) in $B_{R}$ and $B_{R}^{c}$. If $L$ stands again for a bound for the derivative of $f$ in $\left[0,\|u\|_{L^{\infty}\left(\mathbb{R}_{+}^{N}\right)}\right]$, we have

$$
\begin{aligned}
\frac{1}{2}\left\|\widetilde{v}_{\lambda_{j}}\right\|_{L^{\infty}\left(\widetilde{W}_{j}\right)} & \leq\left\|\widetilde{v}_{\lambda_{j}}\right\|_{L^{\infty}\left(\widetilde{W}_{j}\right)}\left(\int_{\Sigma_{\lambda^{*}+1} \cap B_{R}} G_{\infty}^{+}\left(z_{j}, y\right) f^{\prime}\left(\xi_{j}(y)\right) d y\right. \\
& \left.+L \int_{\Sigma_{\lambda^{*}+1} \cap B_{R}^{c}} G_{\infty}^{+}\left(z_{j}, y\right) d y\right) .
\end{aligned}
$$

Observe that the integral in $\Sigma_{\lambda^{*}+1} \cap B_{R}^{c}$ can be made as small as desired by taking $R$ large enough, thanks to the fact that $\left\{z_{j}\right\}$ is a bounded sequence and Lemma 7 part (a). Therefore the last term in the above inequality can be made less than $\frac{1}{4}$, say, if $R$ is chosen large. After we have fixed such a value of $R$, we observe that $\xi_{j} \rightarrow 0$ uniformly in $B_{R}$, so that $f^{\prime}\left(\xi_{j}\right) \leq o(1)$, since we are assuming $f^{\prime}(0) \leq 0$. Therefore, using Lemma 7 , part (b), we arrive at

$$
\frac{1}{4}\left\|\widetilde{v}_{\lambda_{j}}\right\|_{L^{\infty}\left(\widetilde{W}_{j}\right)} \leq o(1)\left\|\widetilde{v}_{\lambda_{j}}\right\|_{L^{\infty}\left(\widetilde{W}_{j}\right)}
$$

which, as above, is a contradiction.

Step 3. Proof of (1.6).

As a consequence of steps 1 and 2 , we have shown that $u^{\lambda} \geq u$ in $\Sigma_{\lambda}$ for every $\lambda>0$, that is, $u$ is nondecreasing as a function of the variable $x_{N}$. Since $u \in C^{1}\left(\mathbb{R}_{+}^{N}\right)$, this implies

$$
\frac{\partial u}{\partial x_{N}} \geq 0 \quad \text { in } \mathbb{R}_{+}^{N}
$$

To conclude the proof of our theorem, we only have to show that the inequality is strict in (5.6). This is a consequence of the strong maximum principle for the derivative with respect to $x_{N}$. However, this function does not directly verify an equation in $\mathbb{R}_{+}^{N}$, since $u$ is not expected to be $C^{1}$ on $\partial \mathbb{R}_{+}^{N}$. We overcome this difficulty by localizing the problem and working with incremental quotients.

Assume there exists $x_{0} \in \mathbb{R}_{+}^{N}$ such that

$$
\frac{\partial u}{\partial x_{N}}\left(x_{0}\right)=0 .
$$

Choose $\delta>0$ such that $B_{2 \delta}\left(x_{0}\right) \subset \subset \mathbb{R}_{+}^{N}$, and let $\phi \in C_{0}^{\infty}\left(B_{2 \delta}\left(x_{0}\right)\right)$ be a cut-off function with the usual properties: $0 \leq \phi \leq 1$ and $\phi=1$ in $B_{\delta}\left(x_{0}\right)$. Choose a small $\tau>0$ and define for $x \in \mathbb{R}^{N}$ :

$$
z_{\tau}(x)=\frac{u\left(x+\tau e_{N}\right)-u(x)}{\tau} \phi(x) .
$$

Since $u$ is nondecreasing we have $z_{\tau} \geq 0$, and we obtain

$$
(-\Delta)^{s} z_{\tau} \geq \frac{f\left(u\left(x+\tau e_{N}\right)\right)-f(u(x))}{\tau} \text { in } B_{\delta}\left(x_{0}\right),
$$

with $z_{\tau} \geq 0$ in $\mathbb{R}^{N}$. Letting $\tau \rightarrow 0$, it is clear that

$$
z_{t} \rightarrow z:=\frac{\partial u}{\partial x_{N}} \phi \quad \text { uniformly in } \mathbb{R}^{N}
$$


and using Lemma 4.5 in [13] we see that

$$
(-\Delta)^{s} z \geq f^{\prime}(u) z \text { in } B_{\delta}\left(x_{0}\right)
$$

in the viscosity sense. The strong maximum principle and (5.7) imply $z=0$ in $B_{\delta}\left(x_{0}\right)$, so that $\frac{\partial u}{\partial x_{N}}=0$ in $B_{\delta}\left(x_{0}\right)$. A standard connectedness argument then implies that

$$
\frac{\partial u}{\partial x_{N}}=0 \text { in } \mathbb{R}_{+}^{N},
$$

which is impossible. This concludes the proof of (1.6). Observe by the way that $u>0$ in $\mathbb{R}_{+}^{N}$ is a direct consequence of (1.6).

Proof of Theorem Q Assume $u$ is a nonnegative, bounded, nontrivial solution of (1.1). By Theorem 1, we have $u>0$ in $\mathbb{R}_{+}^{N}$. We first claim that for every $\delta>0$, there exists $c(\delta)>0$ such that

$$
u(x) \geq c(\delta) \quad \text { if } x_{N} \geq \delta .
$$

Suppose for a contradiction that this is not true. Then there exists $\delta>0$ and a sequence $\left\{x_{n}^{\prime}\right\} \subset \mathbb{R}^{N-1}$ such that $u\left(x_{n}^{\prime}, \delta\right) \rightarrow 0$. Define

$$
u_{n}(x)=u\left(x^{\prime}+x_{n}^{\prime}, x_{N}\right), \quad x \in \mathbb{R}_{+}^{N} .
$$

Since $\left\{u_{n}\right\}$ is uniformly bounded, we obtain after passing to a subsequence that $u_{n} \rightarrow \bar{u}$ locally uniformly in $\mathbb{R}^{N}$, where $\bar{u}$ is a nonnegative, bounded solution of (1.1) which verifies $\bar{u}(0, \delta)=0$. Again by Theorem 1 we see that $\bar{u} \equiv 0$

This is impossible if $f$ verifies (a) in the statement. When $f$ verifies (b), a similar argument as in case (c) in step 2 of the proof of Theorem 1 also leads to a contradiction. This contradiction proves the claim.

Now fix any $\delta>0$ and let

$$
\theta=\inf _{c(\delta) \leq t \leq M} f^{\prime}(t)>0,
$$

where $M=\|u\|_{L^{\infty}\left(\mathbb{R}_{+}^{N}\right)}$. If we choose any function $\phi \in C_{0}^{\infty}\left(\mathbb{R}_{+}^{N} \backslash \Sigma_{\delta}\right)$ such that $0 \leq \phi \leq 1$ and $\phi=1$ in $\mathbb{R}^{N} \backslash \Sigma_{2 \delta}$, we see as in step 3 in the proof of Theorem 1 that the function

$$
z=\frac{\partial u}{\partial x_{N}} \phi
$$

verifies

$$
(-\Delta)^{s} z \geq \theta z \quad \text { in } \mathbb{R}^{N} \backslash \Sigma_{2 \delta} .
$$

Arguing again with the principal eigenvalue $\lambda_{1}(B)$ in a sufficiently large ball $B$ contained in $\mathbb{R}^{N} \backslash \Sigma_{2 \delta}$ we reach a contradiction. This shows that no bounded, nonnegative, nontrivial solution to (1.1) may exist under our hypotheses.

Acknowledgements. B. B. was partially supported by MEC Juan de la Cierva postdoctoral fellowship number FJCI-2014-20504 (Spain). L. D. P. was partially supported by PICT2012 0153 from ANPCyT (Argentina). B. B., J. G-M. and A. Q. were partially supported by Ministerio de Ciencia e Innovación under grant MTM2014-52822-P (Spain). A. Q. was also partially 
supported by Fondecyt Grant No. 1151180 Programa Basal, CMM. U. de Chile and Millennium Nucleus Center for Analysis of PDE NC130017. B. B. and J. G-M. would like to thank the Mathematics Department of Universidad Técnica Federico Santa María where part of this work has been done for its kind hospitality.

\section{REFERENCES}

[1] G.Alberti, G.Bellettini, A nonlocal anisotropic model for phase transitions. I. The optimal profile problem. Math. Ann. 310 (3) (1998), 527-560.

[2] D. Applebaum, "Lévy Processes and Stochastic Calculus", 2nd ed, Cambridge Studies in Advanced Mathematics 116, Cambridge University Press, Cambridge, 2009.

[3] H. Berestycki, L. Caffarelli, L. Nirenberg, Symmetry for elliptic equations in a half-space, pp. 27-42 in: Boundary Value Problems for Partial Differential Equations and Applications, RMA Res. Notes Appl. Math., 29, J. L. Lions et al., eds., Masson, Paris, 1993

[4] H. Berestycki, L. Caffarelli, L. Nirenberg, Inequalities for second-order elliptic equations with applications to unbounded domains I. Duke Math. J. 81 (1996), 467494.

[5] H. Berestycki, L. Caffarelli, L. Nirenberg, Further qualitative properties for elliptic equations in unbounded domains. Ann. Scuola Norm. Sup. Pisa Cl. Sci. (4) 25 (1997), 69-94.

[6] H. Berestycki, L. Caffarelli, L. Nirenberg, Monotonicity for elliptic equations in unbounded Lipschitz domains. Comm. Pure Appl. Math. L (1997), 1089-1111.

[7] J. Bertoin, "Lévy Processes", Cambridge Tracts in Mathematics, 121. Cambridge University Press, Cambridge, 1996.

[8] R. M. Blumenthal, R. K. Getoor, D. B. Ray, On the distribution of the first hits for the symmetric stable processes. Trans. Amer. Math. Soc. 99 (1961), 540-554.

[9] J. P. Bouchaud, A. Georges, Anomalous diffusion in disordered media, Statistical mechanics, models and physical applications, Physics reports 195 (1990).

[10] X. Cabré, J. Sola-Morales, Layer solutions in a half-space for boundary reactions. Comm. Pure Appl. Math. 58 (12) (2005), 1678-1732.

[11] L. CAffarelli, Further regularity for the Signorini problem. Comm. Partial Differential Equations 4 (9) (1979), 1067-1075.

[12] L. Caffarelli, J. M. Roquejoffre, Y. Sire, Variational problems with free boundaries for the fractional Laplacian. J. Eur. Math. Soc. 12 (2010), no. 5, 1151-1179.

[13] L. Caffarelli, L. Silvestre, Regularity theory for fully nonlinear integro differential equations. Comm. Pure Appl. Math. 62 (2009), no. 5, 597-638.

[14] L. Caffarelli, L. Silvestre, Regularity results for nonlocal equations by approximation. Arch. Rat Mech. Anal. 200 (2011), 59-88.

[15] L. Caffarelli, L. VAsseur, Drift diffusion equations with fractional diffusion and the quasi-geostrophic equation, Ann. of Math. (2) 171 (2010), no. 3, 1903-1930.

[16] W. Chen, Y. FAng, R. YAng, Liouville theorems involving the fractional Laplacian on a half space. Adv. Math. 274 (2015), 167-198.

[17] P. Constantin, Euler equations, Navier-Stokes equations and turbulence, in "Mathematical Foundation of Turbulent Viscous Flows", Vol. 1871 of Lecture Notes in Math., Springer, Berlin, 2006.

[18] R. Cont, P. TAnkov, "Financial Modelling with Jump Processes", Chapman \& Hall/CRC Financial Mathematics Series, Boca Raton, Fl, 2004.

[19] C. Cortázar, M. Elgueta, J. García-Melián, Nonnegative solutions of semilinear elliptic equations in half-spaces, J. Math. Pures Appl. 106 (2016), 866-876.

[20] N. DANCER, On the number of positive solutions of weakly non-linear elliptic equations when a parameter is large, Proc. Lond. Math. Soc. 53 (1986) 429-452.

[21] N. Dancer, Some notes on the method of moving planes, Bull. Aust. Math. Soc. 46 (3) (1992) 425-434.

[22] S. Dipierro, A. Figalli, E. VAldinoci, Strongly nonlocal dislocation dynamics in crystals, Comm. Partial Differential Equations 39 (2014), no. 12, 2351-2387. 
[23] S. Dipierro, N. SoAve, E. VAldinoci, On fractional elliptic equations in Lipschitz sets and epigraphs: regularity, monotonicity and rigidity results, to appear in Math. Ann. Available at https://arxiv.org/abs/1604.07755

[24] L. Dupaigne, Y. Sire, A Liouville theorem for non local elliptic equations, in "Symmetry for Elliptic PDEs". A. Farina and E. Valdinoci Eds., Contemp. Math. 528, Amer Math. Soc., 2010.

[25] M. M. FALL, T. Weth, Monotonicity and nonexistence results for some fractional elliptic problems in the half space. Comm. Contemp. Math. 18 (2016), 1550012 (25 pages).

[26] A. FARIna, B. Sciunzi, Qualitative properties and classification of nonnegative solutions to $-\Delta u=f(u)$ in unbounded domains when $f(0)<0$, to appear in Rev. Mat. Iberoam.

[27] A. Farina, N. Soave, Symmetry and uniqueness of nonnegative solutions of some problems in the halfspace, J. Math. Anal. Appl. 403 (2013), 215-233.

[28] P. Felmer, Y. WANG, Radial symmetry of positive solutions to equations involving the fractional laplacian, Comm. Contemp. Math. 16 (2014), 1350023 (24 pages).

[29] A. QuaAs, A. SAlort, A. XIA, Principal eigenvalues of fully nonlinear integro-differential elliptic equations with a drift term. Preprint available at http://arxiv.org/abs/1605.09787

[30] A. QuAAS, A. XIA, Liouville type theorems for nonlinear elliptic equations and systems involving fractional Laplacian in the half space. Calc. Var. Part. Diff. Eqns. 52 (2015), 641-659.

[31] X. Ros-Oton, J. Serra, The Dirichlet problem for the fractional Laplacian: regularity up to the boundary. J. Math. Pures Appl. 101 (2014), 275-302.

[32] O. Savin, E. Valdinoci, Elliptic PDEs with fibered nonlinearities. J. Geom. Anal. 19 (2009), no 2, 420-432.

[33] R. Servadei, E. VAldinoci, Variational methods for non-local operators of elliptic type. Discrete Cont. Dyn. Syst. 33 (2013), 2105-2137.

[34] A. Signorini, Questioni di elasticitá non linearizzata e semilinearizzata, Rendiconti di Matematica e delle sue applicazioni 18 (1959), 95-139.

[35] L. Silvestre, Regularity of the obstacle problem for a fractional power of the Laplace operator. Comm. Pure Appl. Math. 60 (2007), no. 1, 67-112.

[36] Y. SiRE, E. VAldinOCI, Fractional Laplacian phase transitions and boundary reactions: a geometric inequality and a symmetry result. J. Funct. Anal. 256 (6) (2009), $1842-1864$.

[37] V. TARAsov, G. ZASlasvky, Fractional dynamics of systems with long-range interaction, Comm. Nonl. Sci. Numer. Simul. 11 (2006), 885-889.

[38] J. Toland, The Peierls-Nabarro and Benjamin-Ono equations. J. Funct. Anal. 145 (1) (1997), 136-150.

B. BARRIOS

Departamento de Análisis Matemático, Universidad de La Laguna

C/. Astrofísico Francisco SÁnchez s/n, 38200 - La Laguna, SPAiN

E-mail address: bbarrios@ull.es

L. DEL PEZzo

CONICET

Departamento de Matemática, FCEyn uBA

Ciudad Universitaria, Pab I (1428)

Buenos Aires, ARGENTINA.

E-mail address: 1dpezzo@dm.uba.ar 
J. García-Melián

Departamento de Análisis Matemático, Universidad de La Laguna

C/. Astrofísico Francisco SÁnchez s/n, 38200 - La Laguna, SPAiN and

Instituto Universitario de Estudios Avanzados (IUdEA) en Física Atómica, Molecular y Fotónica, Universidad de La Laguna

C/. Astrofísico Francisco SÁnchez s/n, 38200 - La Laguna, SPAIN.

E-mail address: jjgarmel@ull.es

A. QuAas

Departamento de Matemática, Universidad Técnica Federico Santa María Casilla V-110, Avda. España, 1680 - Valparaíso, CHILE.

E-mail address: alexander.quaas@usm.cl 